\title{
Long non-coding RNA NORAD regulates angiogenesis of human umbilical vein endothelial cells via miR-590-3p under hypoxic conditions
}

\author{
XIAOXUE ZHAO, XIUFANG WEI, XUEYING WANG and GUOXIAN QI
}

Department of Geriatrics, The First Hospital of China Medical University, Shenyang, Liaoning 110001, P.R. China

Received September 11, 2019; Accepted March 13, 2020

DOI: $10.3892 / \mathrm{mmr} .2020 .11064$

\begin{abstract}
Dysregulation of angiogenesis can be caused by hypoxia, which may result in severe diseases of the heart, including coronary artery disease. Hypoxia-inducible factor 1 (HIF-1) modulates angiogenesis via the regulation of several angiogenic factors. However, the underlying mechanism of hypoxia-induced angiogenesis remains unknown. In the present study, it was hypothesized that long non-coding RNA (lncRNA) non-coding RNA activated by DNA damage (NORAD) may serve a role in the process of angiogenesis via the regulation of microRNA(miR)-590-3p under hypoxic conditions. The effect of NORAD and miR-590-3p on cell viability and properties associated with angiogenesis, including cell migration and tube formation in human umbilical vein endothelial cells (HUVECs) under hypoxic conditions, were assessed. Potential downstream angiogenic factors of miR-590-3p were also determined by molecular experiments. It was identified that NORAD expression was upregulated and miR-590-3p expression was downregulated in hypoxia-exposed HUVECs, and also in myocardial infarction (MI) left ventricle tissues in mice. Moreover, downregulation of NORAD expression resulted in decreased cell viability and angiogenic capacity, but further knocking down miR-590-3p expression reversed these alterations, resulting in increased cell migration and tube formation in HUVECs under hypoxic conditions for $24 \mathrm{~h}$. It was demonstrated that NORAD overexpression also increased cell vitality and tube-formation capacity. Furthermore, NORAD was identified to bind with miR-590-3p directly, and miR-590-3p was shown to target certain proangiogenic agents, such as vascular endothelial growth factor (VEGF)A, fibroblast growth factor (FGF)1 and FGF2 directly. Therefore, the present results suggested that
\end{abstract}

Correspondence to: Professor Guoxian Qi, Department of Geriatrics, The First Hospital of China Medical University, 155 Nanjing North Street, Shenyang, Liaoning 110001, P.R. China E-mail: guoxian_qi@163.com

Key words: hypoxia, non-coding RNA activated by DNA damage, transcriptional activation, microRNA-590-3p, human umbilical vein endothelial cells
lncRNA NORAD may bind with miR-590-3p to regulate the angiogenic ability of HUVECs via the regulation of several downstream proangiogenic factors under hypoxia. Thus, the lncRNA NORAD/miR-590-3p axis may be a novel regulatory pathway in the angiogenic mechanisms in HUVECs, which highlights a potentially novel perspective for treating ischemia/hypoxia-induced angiogenic diseases.

\section{Introduction}

Angiogenesis is a process in which blood vessels are formed from pre-existing vessels, and is vital for vascularization during embryonic development and vessel formation in various organs and tissues $(1,2)$. Vascular endothelial growth factor (VEGF) has been studied as a fundamental growth factor involved in blood vessel formation (3). Moreover, tight regulation of VEGF expression is required during angiogenesis, but this regulation may be altered by certain physiological signals, particularly in hypoxia $(3,4)$. Hypoxia-inducible factor 1 (HIF-1) exerts its effects on every stage of angiogenesis via activation of various angiogenic factors, such as VEGF, or regulation of proangiogenic chemokines (5-7). VEGF also increases the expression of other proangiogenic factors, including fibroblast growth factor (FGF) (5). FGF1 and FGF2 bind to FGF receptors and serve crucial roles in the angiogenic process (8). Hypoxia is primarily caused by ischemia in vivo, which can result in severe diseases, such as coronary artery diseases (9). Coronary artery diseases are one of the leading causes of mortality worldwide (10). Furthermore, ischemic heart disease results in $>1$ million mortalities worldwide each year, and the morbidity and mortality rates have remained high, particularly in developing countries $(10,11)$. It has been demonstrated that brief continual myocardial ischemia enhances blood vessel formation in the adult heart, resulting in improvement to blood supply and improvement in ischemic areas, which may prevent extended ischemic disorders (12). However, the detailed mechanisms of ischemia/hypoxia-induced angiogenesis are not fully understood.

Long non-coding RNAs (lncRNAs), which are non-coding sequences $>200$ nucleotides in length, are involved in various physiological and pathological processes, and diseases $(13,14)$. In the heart, a conserved lncRNA mechanism, known as the Mhrt-related circuit, participates in chromatin remodelling and prevent myocardial hypertrophy (15). It has been revealed that cardiac apoptosis-related lncRNA affects abnormal mitochondrial fission and apoptosis, which may improve the 
treatment of myocardial infarction (16). Furthermore, lncRNA non-coding RNA activated by DNA damage (NORAD), which is involved in the maintenance of genomic stability, has been identified (17). As its activation is strongly associated with a poor prognosis and survival in breast cancer and pancreatic cancer, and high expression of NORAD is observed in gastric cancer cells and tissues, NORAD is considered to be a potential oncogene (18-20). Additionally, NORAD may serve a role in the development of lung carcinoma by regulating the expression of transforming growth factor- $\beta$ (TGF- $\beta$ ) and its associated phenotype (21). Furthermore, among the family of microRNAs (miRNAs/miRs), which are small non-coding RNAs 22 nucleotides in length, hsa-miR-590-5p has been shown to downregulate the TGF- $\beta$ signalling pathway, resulting in a decreased generation of cardiac cells $(22,23)$. Previous studies have also revealed that hypoxia modulates the activities of abundantly expressed miRNAs by regulating proteins involved in post-transcriptional processes of miRNAs (24).

It has been shown that lncRNAs serve an important role in the regulation of angiogenesis to further affect cancer development or diseases (25). However, the function and mechanisms of the majority of lncRNAs have not been previously determined. It was hypothesized that NORAD may regulate endothelial cell properties via miR-590-3p, and modulated angiogenesis under hypoxic conditions. In the present study, the effects of NORAD and miR-590-3p on angiogenesis in human umbilical vein endothelial cells (HUVECs), and whether miR-590-3p affected NORAD-mediated regulation of endothelial cell activities were investigated.

\section{Materials and methods}

Cell culture and grouping treatment. HUVECs were purchased from the Shanghai Zhong Qiao Xin Zhou Biotechnology Company and cultured in M199 complete medium (Gibco; Thermo Fisher Scientific, Inc.) supplemented with $20 \%$ FBS (Sigma-Aldrich; Merck $\mathrm{KGaA}$ ) at $37^{\circ} \mathrm{C}$ with $5 \% \mathrm{CO}_{2}$. When cells reached $\sim 70 \%$ confluency within 3 passages, cells were divided into two groups (normoxia and hypoxia) for hypoxia treatment in subsequent experiments. Normoxia cells were cultured in $5 \% \mathrm{CO}_{2}$ and $21 \% \mathrm{O}_{2}$ at $37^{\circ} \mathrm{C}$ for $24 \mathrm{~h}$, and cells that underwent hypoxia were incubated in a tri-gas incubator (Shanghai Lishen Scientific Equipment Co., Ltd.) with $5 \% \mathrm{CO}_{2}, 1 \% \mathrm{O}_{2}$ and $94 \% \mathrm{~N}_{2}$ at $37^{\circ} \mathrm{C}$ for $24 \mathrm{~h}$. All subsequent experiments were performed $24 \mathrm{~h}$ after culturing. $293 \mathrm{~T}$ cells were also obtained from the Shanghai Zhong Qiao Xin Zhou Biotechnology Company, and cultured in DMEM (HyClone; GE Healthcare Life Sciences) with $10 \% \mathrm{FBS}$ at $37^{\circ} \mathrm{C}$ with $5 \% \mathrm{CO}_{2}$ for $24 \mathrm{~h}$.

Transfection. Specific short hairpin (sh)RNA against lncRNA NORAD (shNORAD) and control shRNA (shNC1) were obtained from Wanleibio Co.,Ltd.miR-590-3p mimics (5'-UAA UUUUAUGUAUAAGCUAGU-3'), miR-590-3p inhibitor (5'-ACUAGCUUAUACAUAAAAUUA-3') and scrambled control (mimics-NC and inhibitor-NC) were purchased from JTS Scientific Co., Ltd. The overall length of NORAD was 5,378 bp, and the overexpression plasmid pcDNA3.1+ (Clontech Laboratories, Inc.) containing the first 2,000 bp of sequence NORAD (NORAD (N-2000 $-\mathrm{OE}$ ) was established. This sequence was used to ensure the potential binding sequence with miR-590-3p was included, and to avoid the binding site of shNORAD. HUVECs were transfected with 100 pmol of shNORAD (shNC1), miR-590-3p mimics (mimics-NC), NORAD ${ }_{1-2000}$-OE plasmid (empty plasmid/vector) or shNORAD combined with miR-590-3p inhibitor (inhibitor-NC) using Lipofectamine ${ }^{\circledR} 2000$ (Invitrogen; Thermo Fisher Scientific, Inc.) according to the manufacturer protocol. Then, $48 \mathrm{~h}$ after transfection, cells were underwent hypoxia treatment as described above.

Cell Counting Kit-8 (CCK-8) assay. A total of $3 \times 10^{3}$ cells/well HUVECs or transfected HUVECs were plated in 96-well plates with 5 replicates per condition. Following incubation at $37^{\circ} \mathrm{C}$ with $5 \% \mathrm{CO}_{2}$ for $24 \mathrm{~h}$, cells underwent normoxia or hypoxia treatment and were cultured for 12 or $24 \mathrm{~h}$, respectively. Subsequently, $10 \mu$ l CCK-8 reagent (Sigma-Aldrich; Merck KGaA) was added and incubated at $37^{\circ} \mathrm{C}$ for $1 \mathrm{~h}$ according to the manufacturer's instructions. Absorbance was measured at $450 \mathrm{~nm}$ using a microplate reader (BioTek Instruments, Inc.).

Transwell migration assay. Transwell chambers with $8.0 \mu \mathrm{m}$ pore polycarbonate membrane inserts (Corning, Inc.) were placed in 24-well-plates. A total of $4 \times 10^{3}$ normal or transfected HUVECs were suspended in serum-free M199 medium and seeded into the upper chamber, and $800 \mu 1$ medium supplemented with $20 \%$ FBS was added to the lower chamber. Cells were cultured under normoxic or hypoxic conditions at $37^{\circ} \mathrm{C}$ for $24 \mathrm{~h}$ and washed twice with PBS. Cells that had migrated through the filter were fixed with $4 \%$ paraformaldehyde (Sinopharm Chemical Reagent Co., Ltd.) for $20 \mathrm{~min}$ and stained using $0.1 \%$ crystal violet (Amresco, LLC) for $5 \mathrm{~min}$ at room temperature. The stained cells were visualized using an inverted phase contrast microscope (magnification, x200) (Olympus Corporation), and the number of cells in 5 randomly chosen fields was counted.

Tube formation assay. Matrigel (BD Biosciences) was thawed overnight at $4^{\circ} \mathrm{C}$, and used to coat the wells of a 96-well plate (50 $\mu \mathrm{l}$ per well) at $37^{\circ} \mathrm{C}$ for $2 \mathrm{~h}$. Subsequently, HUVECs and transfected HUVECs were suspended and seeded in the

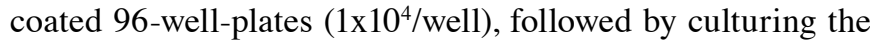
cells under normoxia or hypoxia at $37^{\circ} \mathrm{C}$ for $24 \mathrm{~h}$. Tube formation was observed and imaged using an inverted phase contrast microscope (magnification, x100) (Olympus Corporation), and relative tube length was calculated using Image-Pro Plus version 6 (Media Cybernetics, Inc.). Tube formation was assessed in 3 randomly selected fields of view.

Dual luciferase reporter assay. Potential target sequences between miR-590-3p and IncRNA NORAD, or other target genes, were predicted using the Bielefeld Bioinformatics Server (BiBiServ)-RNAhybrid (26) tool online. Luciferase reporter assays were performed using $293 \mathrm{~T}$ cells. Cells (70\% confluency) were seeded in 12-well-plates with serum-free DMEM medium treatment for $1 \mathrm{~h}$ prior to transfection. The medium in each well was replaced with complete medium and transfection mixture containing: $1.5 \mu \mathrm{g}$ reporter plasmid pmirGLO (GenScript Co., Ltd.), which included the 3' untranslated region (UTR) of the target genes (NORAD, VEGFA, FGF1 and FGF2) with wild-type or mutated binding sequences, and 75 pmol 
synthetic nucleic acid segment in $200 \mu \mathrm{l}$ Opti-minimum essential medium (Gibco; Thermo Fisher Scientific, Inc.) with $9 \mu \mathrm{l}$ Lipofectamine ${ }^{\circledR} 2000$ (Invitrogen; Thermo Fisher Scientific, Inc.). Cells were cultured at $37^{\circ} \mathrm{C}$ for $48 \mathrm{~h}$, and luciferase activity was measured in comparison with Renilla luciferase activity using a dual luciferase detection assay system (Promega Corporation) according to the manufacturer's protocol.

ELISA. ELISA kits for VEGF A (VEGFA; SEA143Hu), FGF1 (SEA032Hu) and FGF2 (CEA551Hu) were used to measure the respective protein levels in the supernatant cell culture of in different groups under hypoxia according to the manufacturer's protocol (Wuhan USCN Business Co., Ltd.).

Reverse transcription-quantitative $(R T-q) P C R$. Total RNA was extracted from HUVECs and tissues using TRIpure reagent (BioTeke Corporation), and RT into cDNA using Super M-MLV reverse transcriptase (BioTeke Corporation), dNTPs, RNase inhibitor (BioTeke Corporation), $5 \mathrm{X}$ reaction buffer, and primers (RT primer for miRNA, Oligo $(\mathrm{dT})_{15}$ for other genes) at $42^{\circ} \mathrm{C}$ for $30 \mathrm{~min}$ and $70^{\circ} \mathrm{C}$ for $10 \mathrm{~min}$ (miRNA) or $42^{\circ} \mathrm{C}$ for $50 \mathrm{~min}$ and $80^{\circ} \mathrm{C}$ for $10 \mathrm{~min}$ (other genes). Subsequently, qPCR was performed using diluted 2x Power Taq PCR MasterMix (BioTeke Corporation) with SYBR-Green (Sigma-Aldrich; Merck $\mathrm{KGaA}$ ). The thermocycling conditions for miRNA were: $94^{\circ} \mathrm{C}$ for $4 \mathrm{~min}, 40$ cycles of $94^{\circ} \mathrm{C}$ for $15 \mathrm{sec}, 60^{\circ} \mathrm{C}$ for $20 \mathrm{sec}$ and $72^{\circ} \mathrm{C}$ for $15 \mathrm{sec}$; for other genes they were: $94^{\circ} \mathrm{C}$ for $5 \mathrm{~min}, 40$ cycles of $94^{\circ} \mathrm{C}$ for $15 \mathrm{sec}, 60^{\circ} \mathrm{C}$ for $25 \mathrm{sec}$ and $72^{\circ} \mathrm{C}$ for $30 \mathrm{sec}$. The expression of hsa-miR-590-3p was normalized to $5 \mathrm{~S}$, and the rest of the genes was normalized to $\beta$-actin. Primers were purchased from GenScript and the sequences were: hsa-miR-590-3p/mmu-miR-590-3p forward, 5'-TAATTT TATGTATAAGCTAGT-3'; hsa-miR-590-3p/mmu-miR-590-3p reverse, 5'-GCAGGGTCCGAGGTATTC-3'; 5S forward,5'-GAT CTCGGAAGCTAAGCAGG-3'; 5S reverse, 5'-TGGTGCAGG GTCCGAGGTAT-3'; mmu-5S forward, 5'-CTAAAGATTTCC GTGGAGAG-3'; mmu-5S reverse, 5'-TGGTGCAGGGTC CGAGGTAT-3'; IncRNA NORAD forward, 5'-GGAGAATCG CTTGAACT-3'; lncRNA NORAD reverse, 5'-CAAACACCC AATGAATAG-3'; mmu-lncRNA NORAD forward, 5'-GAT TGCCGACGCAGGGTA-3'; mmu-lncRNA NORAD reverse, 5'-CTGAACAAACAGGGACGA-3'; HIF-1 $\alpha$ forward, 5'-GAA ACTTCTGGATGCTGGTG-3'; HIF-1 $\alpha$ reverse, 5'-CAAACT GAGTTAATCCCATG-3'; VEGFA forward, 5'-TCACCAAGG CCAGCACATAG-3'; VEGFA reverse, 5'-GGGCACCAACGT ACACGCT-3'; FGF1 forward, 5'-GAGCGACCAGCACATTCA G-3'; FGF1 reverse, 5'-TCTCCTCCAGCCTTTCCAG-3'; FGF2 forward, 5'-AGAAGAGCGACCCTCACAT-3'; FGF2 reverse, 5'-AAAGAAACACTCATCCGTAA-3'; $\beta$-actin forward, 5'-GGCACCCAGCACAATGAA-3'; $\beta$-actin reverse, 5'-TAG AAGCATTTGCGGTGG-3'; mmu- $\beta$-actin forward, 5'-CTG TGCCCATCTACGAGGGCTAT- 3 ; and mmu- $\beta$-actin reverse, 5'-TTTGATGTCACGCACGATTTCC-3'. Fold changes of gene expressions were calculated using the $2^{-\Delta \Delta C q}$ method (27).

Western blotting. Cell Lysis Buffer for Western or IP (Beyotime Institute of Biotechnology) was used to extract the total protein. Protein concentration was detected using a bicinchoninic acid protein assay kit (Beyotime Institute of Biotechnology). A total of 20-40 $\mu \mathrm{g}$ was loaded on an $8-12 \%$
SDS gel and resolved using SDS-PAGE. The resolved proteins were transferred to PVDF membranes (EMD Millipore) and were blocked in 5\% non-fat milk (Yili Group) at room temperature for $1 \mathrm{~h}$. Membranes were incubated with primary antibodies against HIF-1 $\alpha$ (AF1009, Affinity Biosciences) and $\beta$-actin (sc-47778; Santa Cruz Biotechnology, Inc.) both at $1: 1,000$ at $4^{\circ} \mathrm{C}$ overnight. Incubation of membranes with horseradish peroxidase-conjugated goat anti-rabbit (A0208, 1:5,000; Beyotime) or anti-mouse (A0216, 1:5,000; Beyotime) secondary antibody was performed at $37^{\circ} \mathrm{C}$ for $45 \mathrm{~min}$. An ECL reagent (Beyotime Institute of Biotechnology) was used to visualize the signals, and densitometry analysis was performed using Gel-Pro-Analyzer software (version 4; Media Cybernetics, Inc.).

Animal experiment. A total of 20 male C57 mice (8 weeks old, 22 \pm 2 g; Beijing HFK Bioscience Co., Ltd.) were used to establish a myocardial infarction (MI) model via left anterior descending (LAD) ligation surgery. Prior to surgery, all mice were maintained in a $12 \mathrm{~h}$ light/dark cycle with constant temperature of $22 \pm 1^{\circ} \mathrm{C}$ and $45-55 \%$ humidity, with free access to food and water. After week, mice were immobilized and anesthetized. Then, endotracheal intubation and ventilation were performed following incision of the neck trachea, followed by left thoracotomy between the third and fourth intercostal space. Blood flow in the anterior descending branch was blocked using a sterile $8 / 0$ suture ligation. Mice in the control group $(n=6)$ underwent the same operation, but without ligation. After $24 \mathrm{~h}$ following suture, the left ventricle tissues were removed for analysis. Each group contained six mice. Animal experiments were performed in accordance with the principles described in the Guide for The Care and Use of Laboratory Animals (28), and were approved by The Ethics Committee of The First Hospital of China Medical University.

Statistical analysis. Data were presented as the mean \pm SD of 3 repeats for the in vitro experiments, and for the animal experiments there were 6 animals per group. GraphPad Prism version 8 (GraphPad Prism Software, Inc.) was used to perform the statistical analysis. Unpaired t-tests were used to compare two independent groups, and differences between $\geq 3$ groups were compared using the one-way ANOVA combined with Tukey's multiple comparisons post hoc test. $\mathrm{P}<0.05$ was considered to indicate a statistically significant difference.

\section{Results}

HUVEC tube-formation ability is increased under hypoxic conditions. To determine the effects of non-coding RNAs on hypoxia exposed HUVECs, experiments were performed to assess the activities of HUVECs under hypoxic conditions. mRNA expression levels of HIF-1 $\alpha$ and its downstream target VEGFA were determined $24 \mathrm{~h}$ after hypoxia culturing using RT-qPCR, and it was identified that both had increased expression levels following hypoxia treatment (Fig. 1A). Western blotting results also demonstrated increased protein expression of HIF-1 $\alpha$ under hypoxic conditions (Fig. 1B). Thus, these results suggested that hypoxia treatment was effective. Furthermore, the CCK- 8 assay results indicated that, compared with cells in the normoxic condition, cell viability 
A

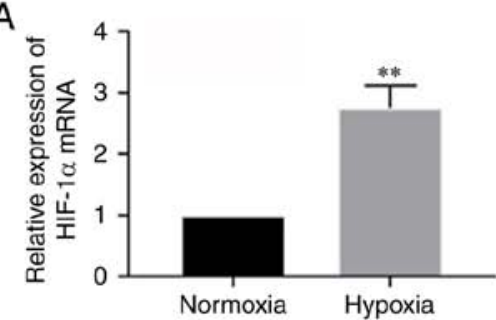

C

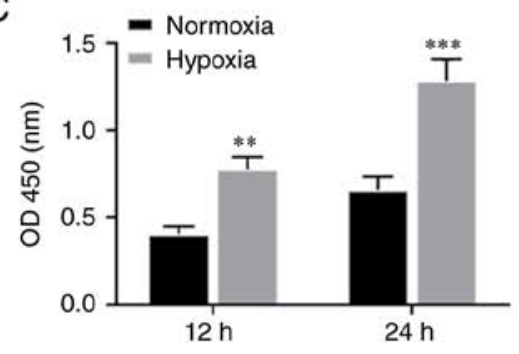

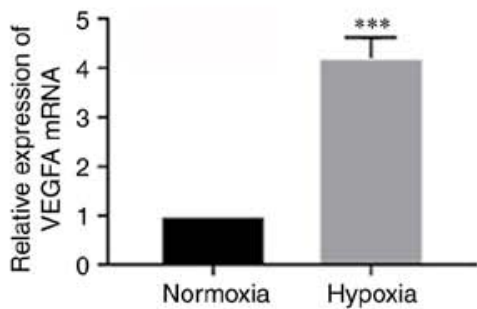

D

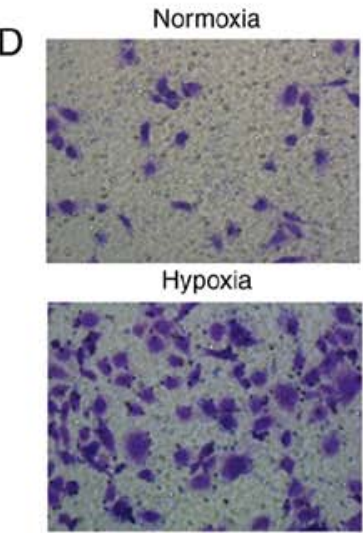

B

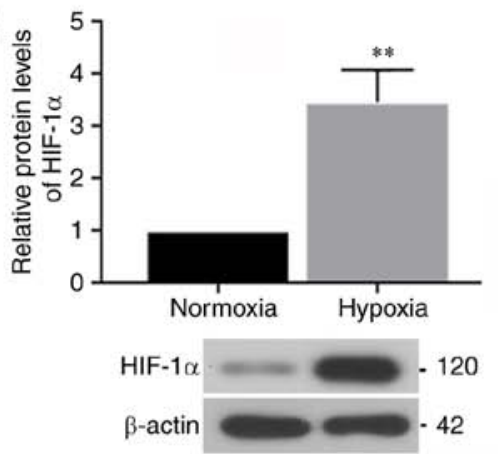

$\mathrm{E}$

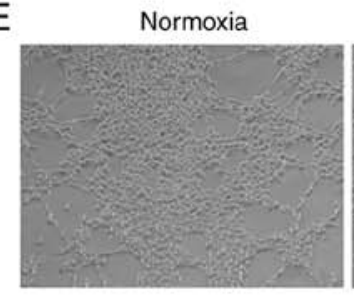

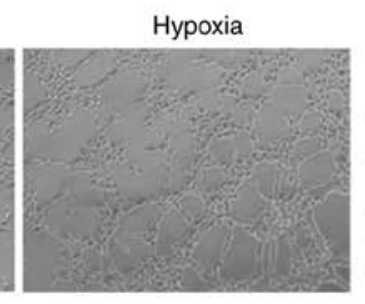
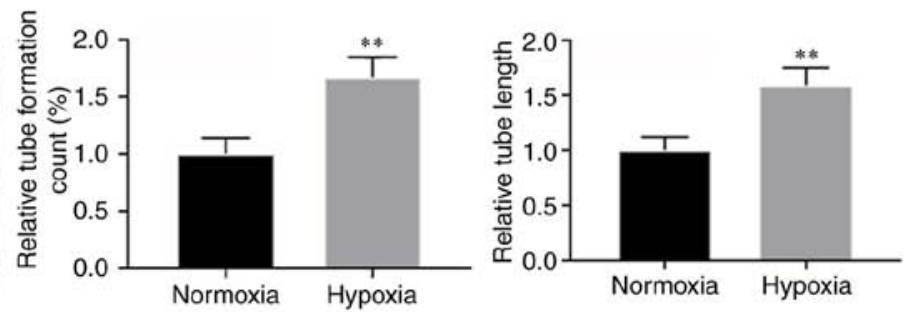

Figure 1. HUVEC tube-forming ability is increased under hypoxic conditions. (A) mRNA expression levels of HIF-1 $\alpha$ and its target, VEGFA, under normoxic and hypoxic conditions. (B) Protein expression of HIF-1 $\alpha$ following hypoxic culturing for $24 \mathrm{~h}$. (C) Cell viability of HUVECs following normoxia and hypoxia treatment for 12 or $24 \mathrm{~h}$, respectively. (D) Transwell migration assay was performed to assess the migratory capacity of cells under normoxic and hypoxic conditions. Representative images of migration in both cells. Magnification, x200. (E) Tube formation ability of HUVECs under normoxia and hypoxia. Representative images and quantification of tube count and length. Magnification, $\mathrm{x} 100 .{ }^{* * *} \mathrm{P}<0.01$ and ${ }^{* * *} \mathrm{P}<0.001$ vs. normoxia. HIF-1 $\alpha$, hypoxia-inducible factor-1 $\alpha$; VEGFA, vascular endothelial growth factor A; OD, optical density.

of HUVECs was increased after $24 \mathrm{~h}$ of hypoxia exposure (Fig. 1C). Moreover, Transwell assays were performed to assess the migratory ability of cells following hypoxia for $24 \mathrm{~h}$, and it was identified that hypoxia significantly increased migration (Fig. 1D). Furthermore, results from the tube formation assay in the hypoxia-exposed HUVECs demonstrated that tube count and length were increased compared with normoxic cells (Fig. 1E). Therefore, the present results suggested that short-term hypoxia exposure caused increased tube-formation in HUVECs.

IncRNA NORAD expression is increased and miR-590-3p expression is decreased in hypoxic HUVECs in vitro and MI left ventricular tissues in vivo. The relative mRNA expression levels of lncRNA NORAD and miR-590-3p in HUVECs under hypoxia were assessed using RT-qPCR. It was demonstrated that NORAD expression was increased and miR-590-3p expression was decreased in HUVECs exposed to hypoxia for $24 \mathrm{~h}$ (Fig. 2A). In vivo, mice MI models were also used to assess the expression levels of these 2 factors in the left ventricle following MI modelling for $24 \mathrm{~h}$, and similar results were observed as those identified in vitro (Fig 2B).
Thus, it was identified that hypoxia increased expression of IncRNA NORAD, and decreased expression of miR-590-3p, in HUVECs in vitro and in left ventricular tissues in vivo.

IncRNA NORAD knockdown inhibits tube-formation ability of HUVECs under hypoxia. To investigate how lncRNA NORAD affected the HUVECs under hypoxia, cells were transfected with shNORAD and shNC1. RT-qPCR was performed to detect the relative expression of NORAD in transfected HUVECs, and it was revealed that shNORAD effectively knocked down the expression of IncRNA NORAD in HUVECs (Fig. 3A). Moreover, hypoxic treatment for $24 \mathrm{~h}$ in shNORAD transfected HUVECs also demonstrated that NORAD expression was significantly knocked down. It was also identified that NORAD downregulation caused an increase in miR-590-3p expression under hypoxia (Fig. 3B). Cell viability of transfected HUVECs was assessed using a CCK-8 assay. In contrast to hypoxia-exposed shNC1 cells, knockdown of NORAD resulted in decreased viability of shNORAD transfected HUVECs (Fig. 3C). Furthermore, the Transwell migration assays showed that HUVECs transfected with shNORAD exhibited a lower migratory 
A
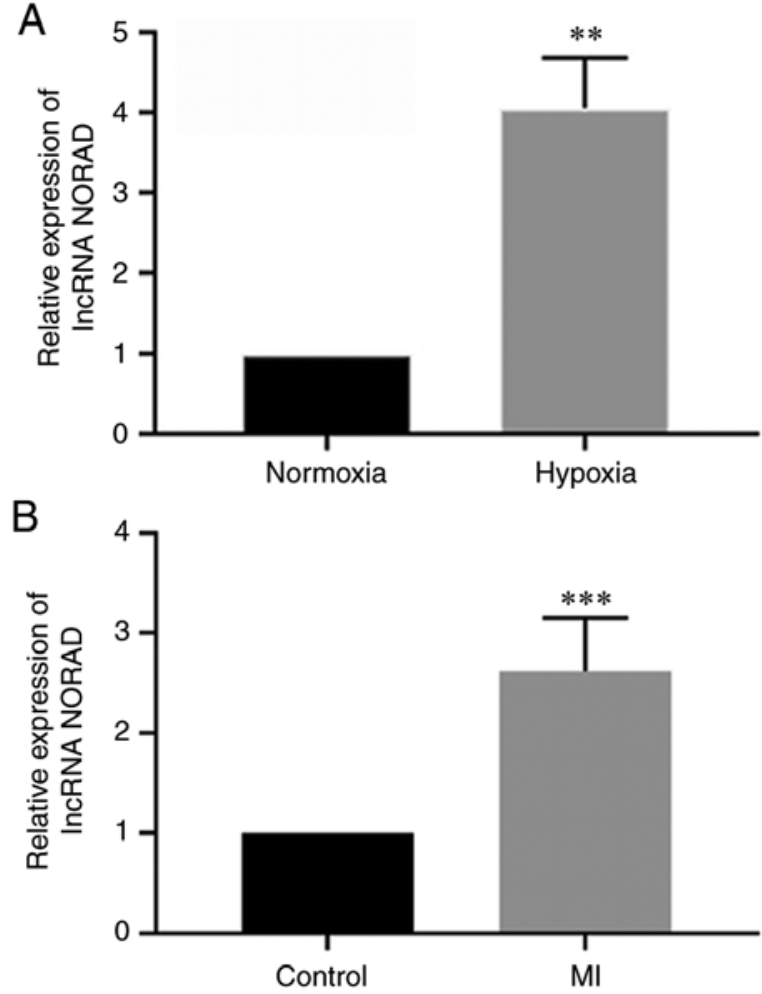
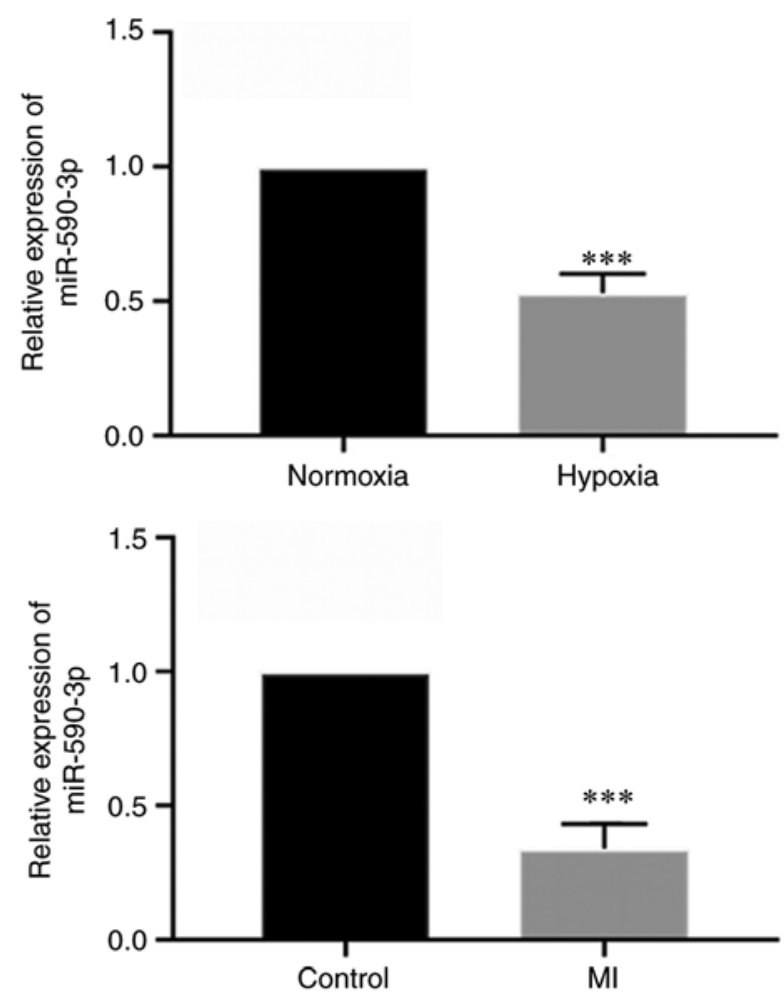

Figure 2. IncRNA NORAD expression is increased and miR-590-3p expression is decreased in hypoxic HUVECs in vitro and MI left ventricular tissues in vivo. mRNA expression levels of lncRNA NORAD and miR-590-3p in (A) HUVECs under normoxic and hypoxic conditions, and (B) mice left ventricle tissues after $24 \mathrm{~h}$ of MI modelling. ${ }^{* *} \mathrm{P}<0.01$ and ${ }^{* * *} \mathrm{P}<0.001$ vs. normoxia or control. MI, myocardial infarction; lncRNA, long non-coding RNA; lncRNA NORAD, lncRNA non-coding RNA activated by DNA damage; miR, microRNA.

capacity compared with cells transfected with shNC1 under hypoxia (Fig. 3D). NORAD knockdown also decreased tube formation ability of HUVECs exposed to hypoxia compared with the NC cells (Fig. 3E). Therefore, the present results suggested that lncRNA NORAD knockdown inhibited the angiogenesis-associated abilities of HUVECs under hypoxia, indicating decreased blood vessel formation.

IncRNA NORAD overexpression promotes tube-formation in HUVECs under hypoxia. Cells were transfected with the NORAD $D_{1-2000}-\mathrm{OE}$ plasmid to study the effects of NORAD overexpression on HUVECs under hypoxia. Under normoxic conditions, the transfection of NORAD ${ }_{1-2000}-\mathrm{OE}$ plasmid in HUVECs was highly efficient (Fig. 4A). Moreover, the relative expression of NORAD was significantly upregulated, and the expression of miR-590-3p was decreased in the NORAD ${ }_{1-2000}-\mathrm{OE}$ transfected cells under hypoxia (Fig. 4B). Cell viability, migratory capacity and tube formation, including tube count and length, were assessed in NORAD overexpressed cells under hypoxia, and were identified to be increased (Fig. 4C-E). Collectively, the present results indicated that NORAD overexpression in HUVECs reversed the reduced tube-formation ability caused by NORAD knockdown under hypoxia.

Overexpression of miR-590-3p suppresses tube formation in HUVECs under hypoxia. To determine the role of miR-590-3p in the vasculogenic ability of HUVECs under hypoxia, cells were transfected with miR-590-3p mimics and mimics-NC. The RT-qPCR results indicated that miR-590-3p was efficiently overexpressed when cells were transfected with miR-590-3p mimics, under both normoxic and hypoxic conditions (Fig. 5A). Furthermore, overexpression of miR-590-3p resulted in similar effects on cell viability, cell migration and tube formation ability as knockdown of NORAD under hypoxia (Fig. 5B-D). In the dual luciferase assay, 293T cells co-transfected with NORAD-wild-type and miR-590-3p mimics exhibited significantly decreased luciferase activity compared with cells transfected with mutant NORAD and miR-590-3p mimics (Fig. 5E), which suggested that NORAD could bind with miR-590-3p directly. Thus, it was hypothesized that miR-590-3p also affected HUVEC tube-forming ability under hypoxia, which may be associated with NORAD targeting.

IncRNA NORAD affects angiogenic characteristics of HUVECs under hypoxia via miR-590-3p. To investigate whether the effects of NORAD on HUVEC tube-formation ability were regulated by miR-590-3p under hypoxia, HUVECs were co-transfected with shNORAD and miR-590-3p inhibitor. The transfection efficiency of the miR-590-3p inhibitor under normoxic conditions was assessed using RT-qPCR, which identified that the expression of miR-590-3p was significantly downregulated (Fig. 6A). Subsequently, the relative expression levels of lncRNA NORAD and miR-590-3p were assessed using RT-qPCR following co-transfection. Cells co-transfected with shNORAD and miR-590-3p inhibitor exhibited decreased miR-590-3p expression levels, but increased NORAD expression, compared with cells transfected with shNORAD and inhibitor-NC (Fig. 6B). Therefore, it was 

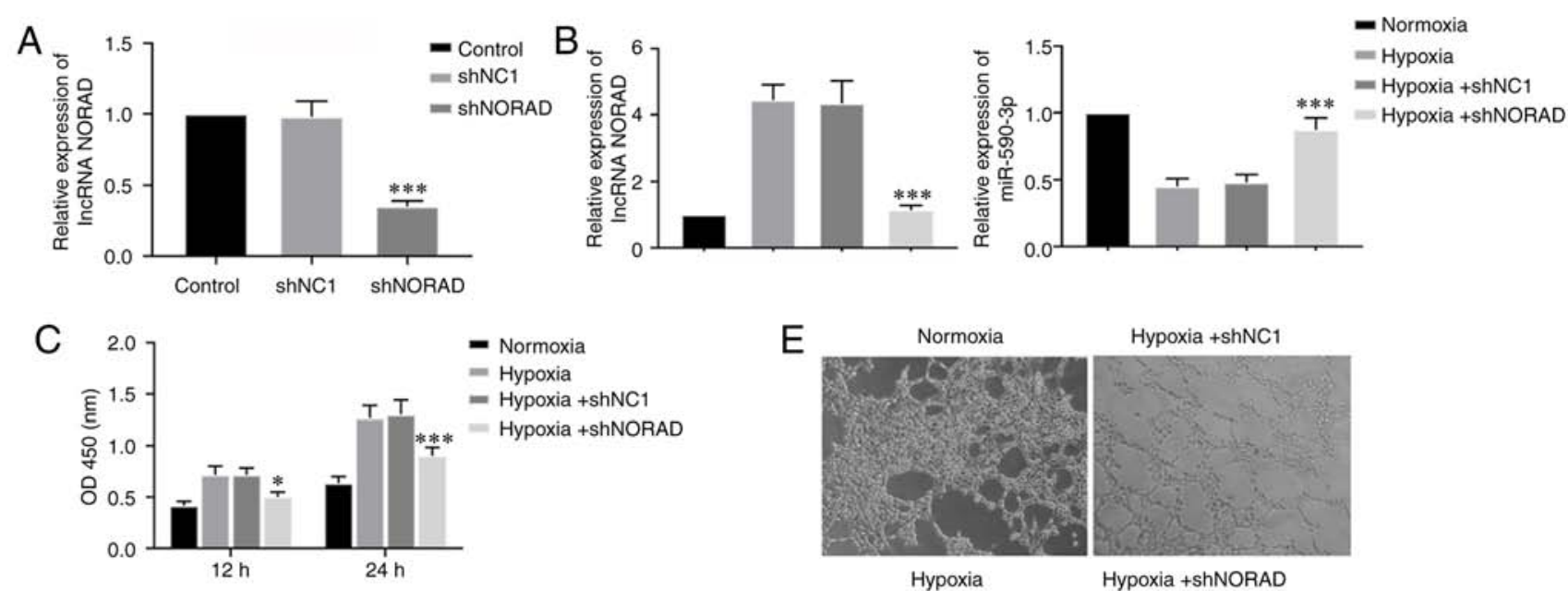
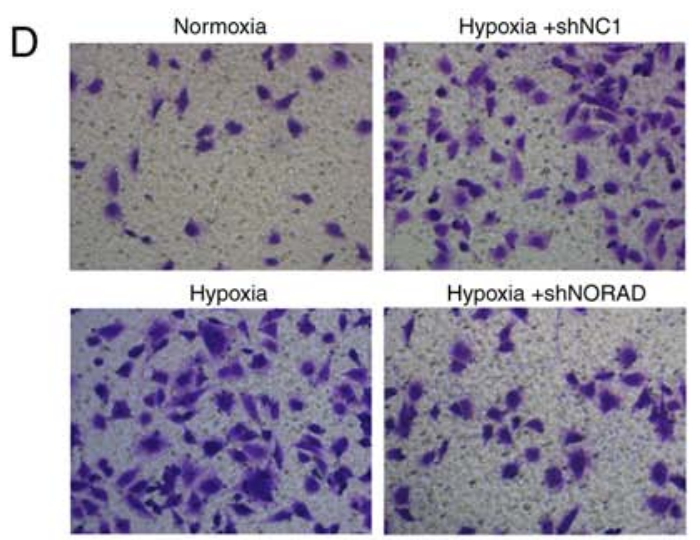

Hypoxia +shNORAD
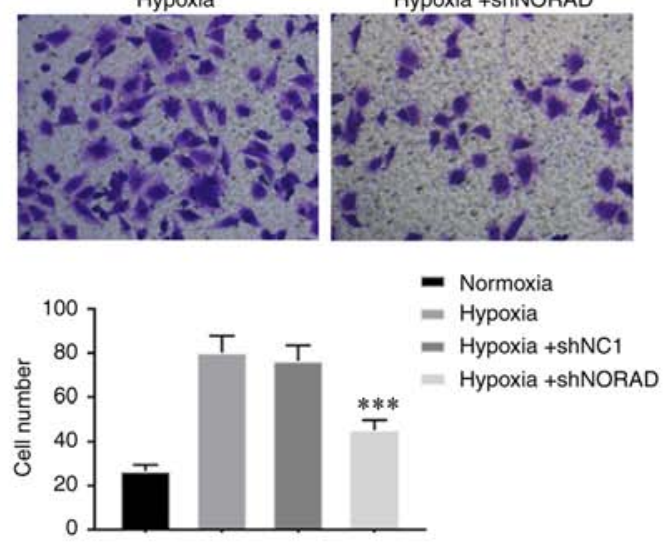
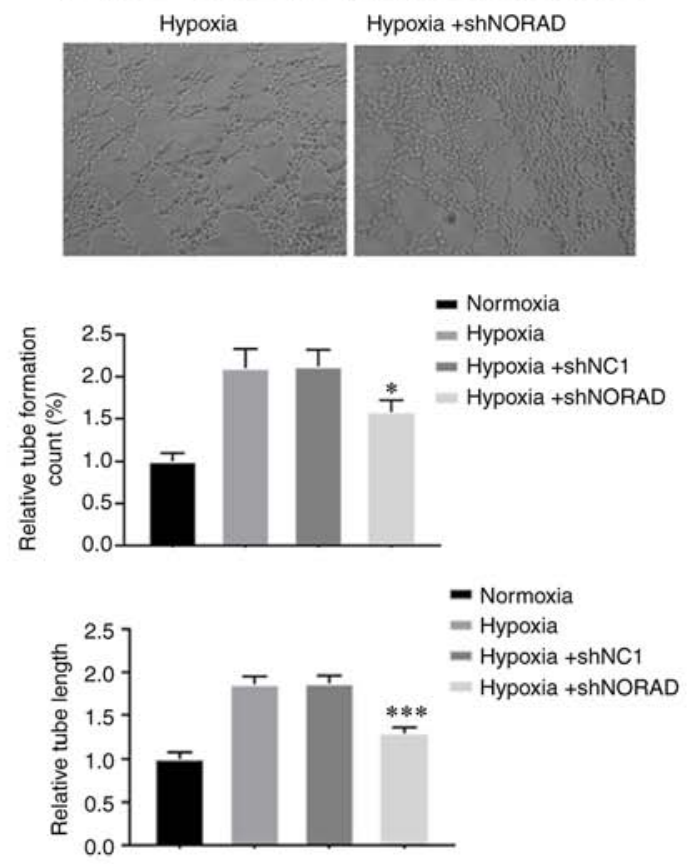

Figure 3. IncRNA NORAD knockdown decreases tube formation in HUVECs under hypoxia. (A) Relative expression of lncRNA NORAD in HUVECs following transfection with shNORAD and shNC1. ${ }^{* * *} \mathrm{P}<0.001$ vs. shNC1. (B) mRNA expression levels of NORAD and miR-590-3p in HUVECs transfected with shNORAD under hypoxic conditions. ${ }^{* * *} \mathrm{P}<0.001$ vs. hypoxia + shNC1. (C) Cell viability of HUVECs transfected with shNORAD under normoxia or hypoxia. ${ }^{*} \mathrm{P}<0.05$ and ${ }^{* * *} \mathrm{P}<0.001$ vs. hypoxia + shNC1. (D) Representative images and quantification of migration in HUVECs transfected with shNORAD under hypoxia. Magnification, $\mathrm{x} 200{ }^{* * * *} \mathrm{P}<0.001 \mathrm{vs}$. hypoxia + shNC1. (E) Representative images and quantification of tube formation of HUVECs transfected with shNORAD under hypoxic conditions. Magnification, $x 100$. ${ }^{*} \mathrm{P}<0.05$ and ${ }^{* * * *} \mathrm{P}<0.001$ vs. hypoxia + shNC1. lncRNA, long non-coding RNA; lncRNA NORAD, lncRNA non-coding RNA activated by DNA damage; miR, microRNA; sh, short hairpin RNA; NC, negative control; OD, optical density.

speculated that miR-590-3p negatively regulated NORAD, and similar results were observed under hypoxia (Fig. 6C). Cell viability in the transfected hypoxia-exposed HUVECs, in which NORAD and miR-590-3p expression levels were downregulated, was significantly increased compared with cells transfected with shNORAD and inhibitor-NC (Fig. 6D). Furthermore, downregulation of NORAD and miR-590-3p in the HUVECs also increased migration and tube formation under hypoxia compared with the control cells (Fig. 6E and F). Thus, downregulation of both NORAD and miR-590-3p in HUVECs reversed the decrease in angiogenic activity caused by knockdown of NORAD alone, which resulted in enhanced angiogenic properties under hypoxic conditions. Collectively, the present results suggested that miR-590-3p lay downstream of the regulatory effects of lncRNA NORAD on angiogenic activity in hypoxia-exposed HUVECs.
Downstream targets of the lncRNA NORAD/miR-590-3p axis in HUVECs under hypoxia. In total, 3 possible regulated genes were proposed, VEGFA, FGF1 and FGF2, all of which are closely associated with angiogenesis and cell proliferation $(5,8)$. After $24 \mathrm{~h}$ of hypoxia treatment, it was demonstrated that the mRNA expression levels of all 3 genes were significantly decreased in NORAD knockdown HUVECs, but significantly increased in cells where NORAD and miR-590-3p were both downregulated (Fig. 7A). Moreover, ELISAs were performed to assess the changes in the levels of all 3 proteins, and it was identified that when NORAD was downregulated, the concentrations of VEGFA, FGF1 and FGF2 were decreased under hypoxia. However, when miR-590-3p was knocked down, the expression levels of these genes were increased significantly under the same condition (Fig. 7B). Binding of miR-590-3p to these genes was determined using a dual 
A

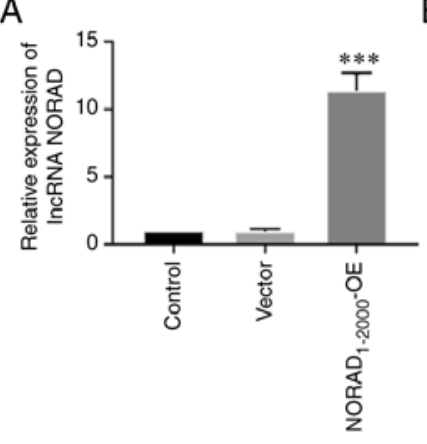

B

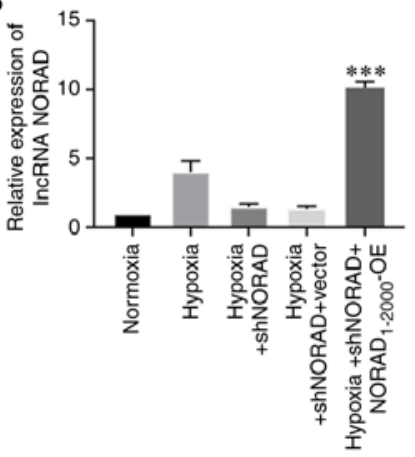

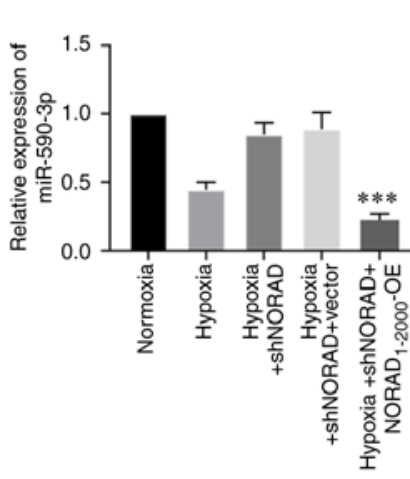

C

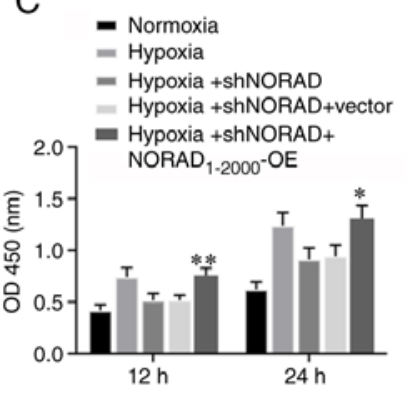

D
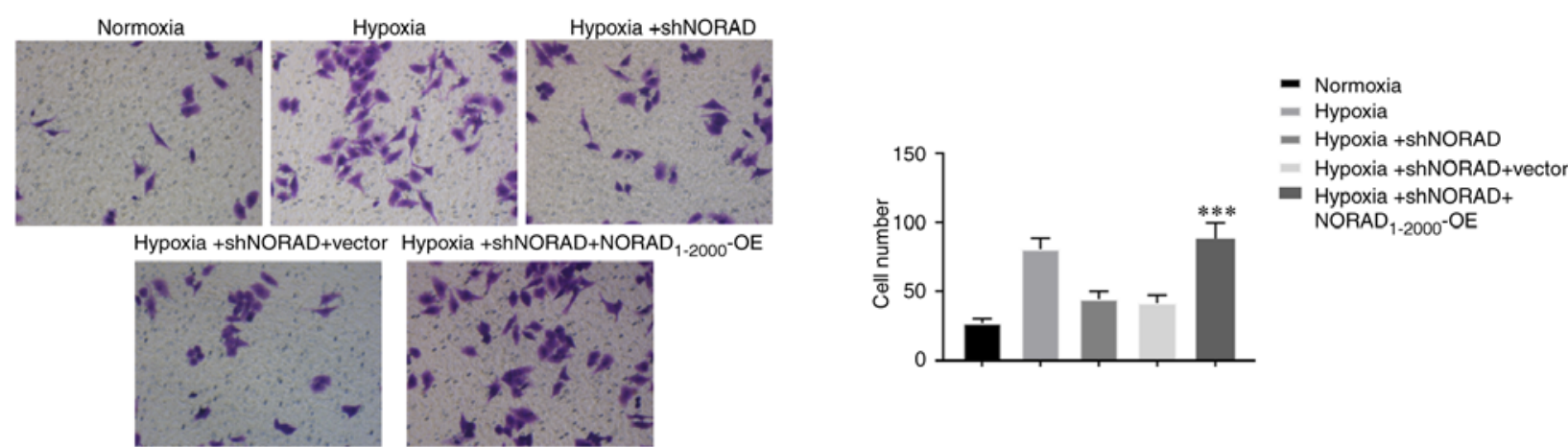

E
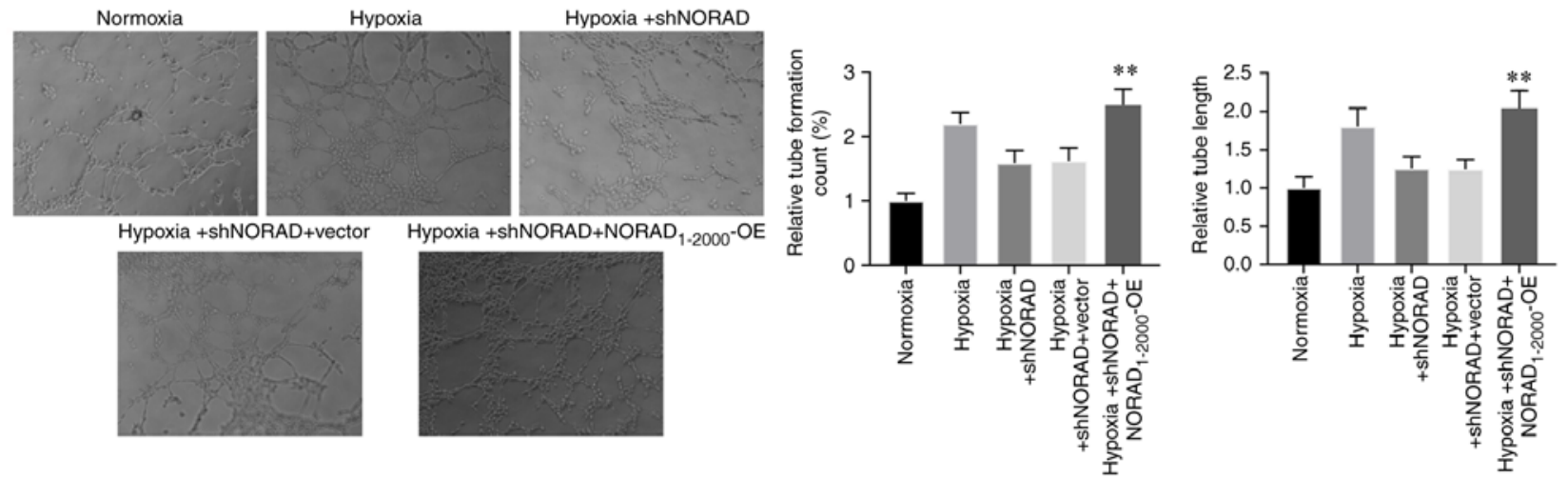

Figure 4. IncRNA NORAD overexpression increases tube formation in HUVECs under hypoxia. (A) Relative expression of lncRNA NORAD in HUVECs after transfection with NORAD ${ }_{1-2000}$-OE plasmid and empty vector. ${ }^{* * * *} \mathrm{P}<0.001$ vs. vector. (B) mRNA expression levels of NORAD and miR-590-3p following overexpression of NORAD under hypoxic conditions. (C) Cell viability, (D) cell migration (magnification, $\mathrm{x} 200$ ) and (E) tube formation (magnification, $\mathrm{x} 100)$ were assessed $24 \mathrm{~h}$ after hypoxia treatment in the NORAD-overexpressing HUVECs. ${ }^{*} \mathrm{P}<0.05,{ }^{* *} \mathrm{P}<0.01$ and ${ }^{* * * *} \mathrm{P}<0.001$ vs. Hypoxia + shNORAD + vector. sh, short hairpin RNA; IncRNA, long non-coding RNA; IncRNA NORAD, IncRNA non-coding RNA activated by DNA damage; miR, microRNA; OD, optical density.

luciferase assay, which identified that cells co-transfected with the 3'UTR of VEGFA, FGF1 or FGF2, and miR-590-3p mimics, exhibited lower luciferase activity compared with cells transfected with their mutated 3'UTR and miR-590-3p mimics (Fig. 7C). Therefore, the present results suggested that a lncRNA NORAD/miR-590-3p axis regulated the activity of the angiogenic indicators VEGFA, FGF1 and FGF2, and that these genes were direct downstream targets of miR-590-3p.

\section{Discussion}

The aim of the present study was to determine whether the lncRNA NORAD served a role in angiogenesis induced by hypoxia, and the involvement of miR-590-3p in this process. lncRNA NORAD is activated by DNA damage and maintains genomic stability via isolating Pumilio proteins, which bind with mRNAs to suppress their stability and translation activity (17). Thus, NORAD is crucial for cell division and growth during cell development (29). In the present study, in short term hypoxia (24 h), downregulation of NORAD in the HUVECs resulted in decreased angiogenic properties, including cell viability, cell migration and tube formation. Moreover, NORAD overexpression resulted in opposite effects, suggesting that NORAD regulates the angiogenic properties of HUVECs under hypoxic conditions. It was identified that the overexpression of miR-590-3p caused a similar decrease in tube formation in hypoxia-exposed HUVECs. The present results observed in cells overexpressing miR-590-3p were consistent with the effects of NORAD downregulation, and when NORAD was downregulated, miR-590-3p expression was increased both in vitro and in vivo. Several miRNAs, including miR-20, miR-130 family and miR-199, are involved in angiogenesis under hypoxic conditions by modulating the expression or activity of proteins, including HIF-1 and 

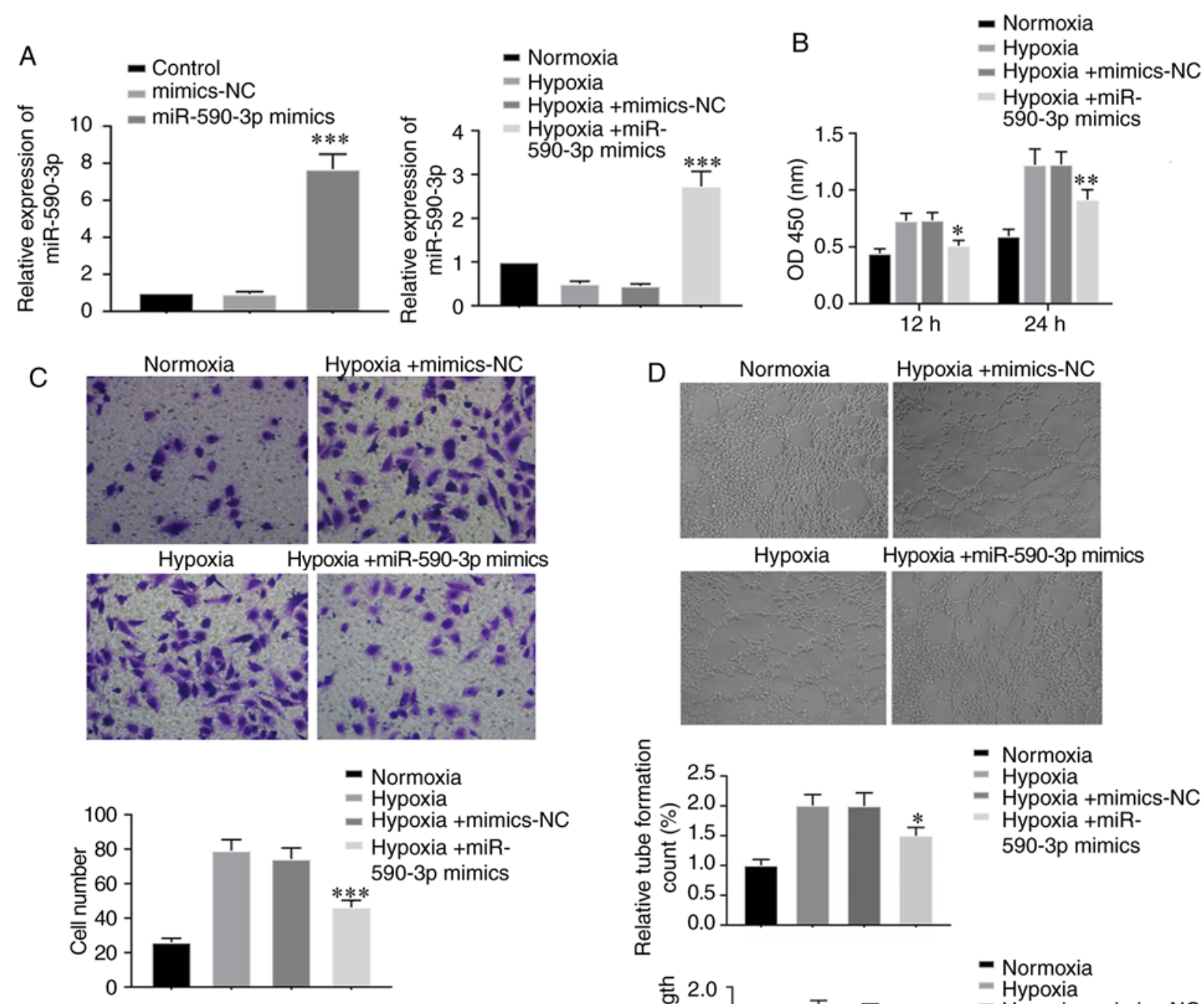

- Normoxia

- Hypoxia

- Hypoxia +mimics-NC

Hypoxia +miR590-3p mimics

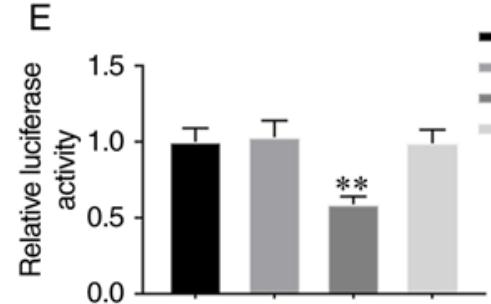

- NORAD-wt +mimics-NC

- NORAD-mut +mimics-NC

NORAD-wt +miR-590-3p mimics

- NORAD-mut+miR-

590-3p mimics

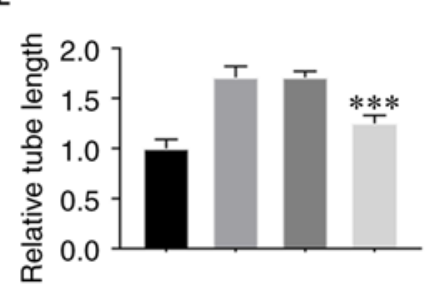
- Normoxia
- Hypoxia
- Hypoxia +mimics-NC
Hypoxia +miR- 590-3p mimics

InCRNA NORAD-mut:

\begin{tabular}{|c|c|}
\hline $\begin{array}{l}\text { Hsa-miR-590-3p: } \\
\text { IncRNA NORAD-wt: }\end{array}$ & 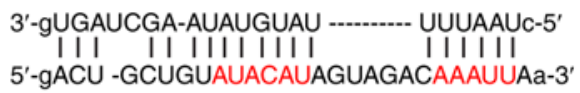 \\
\hline NA NORAD & JAU \\
\hline
\end{tabular}

Figure 5. Overexpression of miR-590-3p suppresses tube-formation in HUVECs under hypoxia. (A) HUVECs were transfected with miR-590-3p mimics and mimics-NC. Relative expression of miR-590-3p following transfection was assessed prior and subsequent to hypoxia treatment. ${ }^{* * * *} \mathrm{P}<0.001 \mathrm{vs}$. respective NC controls. (B) Cell viability, (C) cell migration (magnification, x200) and (D) tube formation (magnification 100x) were examined in cells transfected with miR-590-3p mimics under hypoxia. ${ }^{*} \mathrm{P}<0.05,{ }^{* *} \mathrm{P}<0.01$ and ${ }^{* * *} \mathrm{P}<0.001$ vs. Hypoxia + mimics-NC group. (E) Relative luciferase activity was measured $48 \mathrm{~h}$ after co-transfection of miR-590-3p mimics with NORAD-wt or NORAD-mut in 293T cells. The binding sequences of the wt and mut NORAD are shown. ${ }^{* *} \mathrm{P}<0.01$ vs. NORAD-mut + miR-590-3p mimics group. IncRNA, long non-coding RNA; IncRNA NORAD, IncRNA non-coding RNA activated by DNA damage; miR, microRNA; NC, negative control; mut, mutant; wt, wild-type; OD, optical density.

VEGF (30). Based on the present results, it was speculated that miR-590-3p may also be considered an additional miRNA involved in angiogenesis under hypoxic conditions.

The present results suggested that NORAD directly binds to miR-590-3p, and miR-590-3p directly targeted the 3'UTR of VEGFA, FGF1 and FGF2. In addition, knockdown of both NORAD and miR-590-3p could alleviate and reverse the decreased angiogenic activities and expression levels of proangiogenic indicators caused by knockdown of NORAD alone, leading to increased cell viability, migration, tube formation and expression of associated proangiogenic factors. Collectively, the present results indicated the presence of a regulatory pathway in which lncRNA NORAD binds to miR-590-3p to regulate angiogenesis in HUVECs, by affecting the expression levels of downstream proangiogenic proteins, such as VEGFA, FGF1 and FGF2, under hypoxic conditions. It is possible that other positive factors involved in the regulation of angiogenesis also serve a role in the NORAD/miR-590-3p 


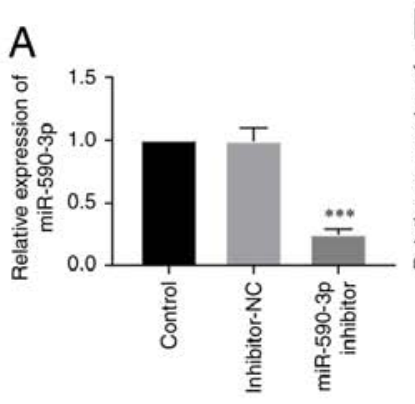

B
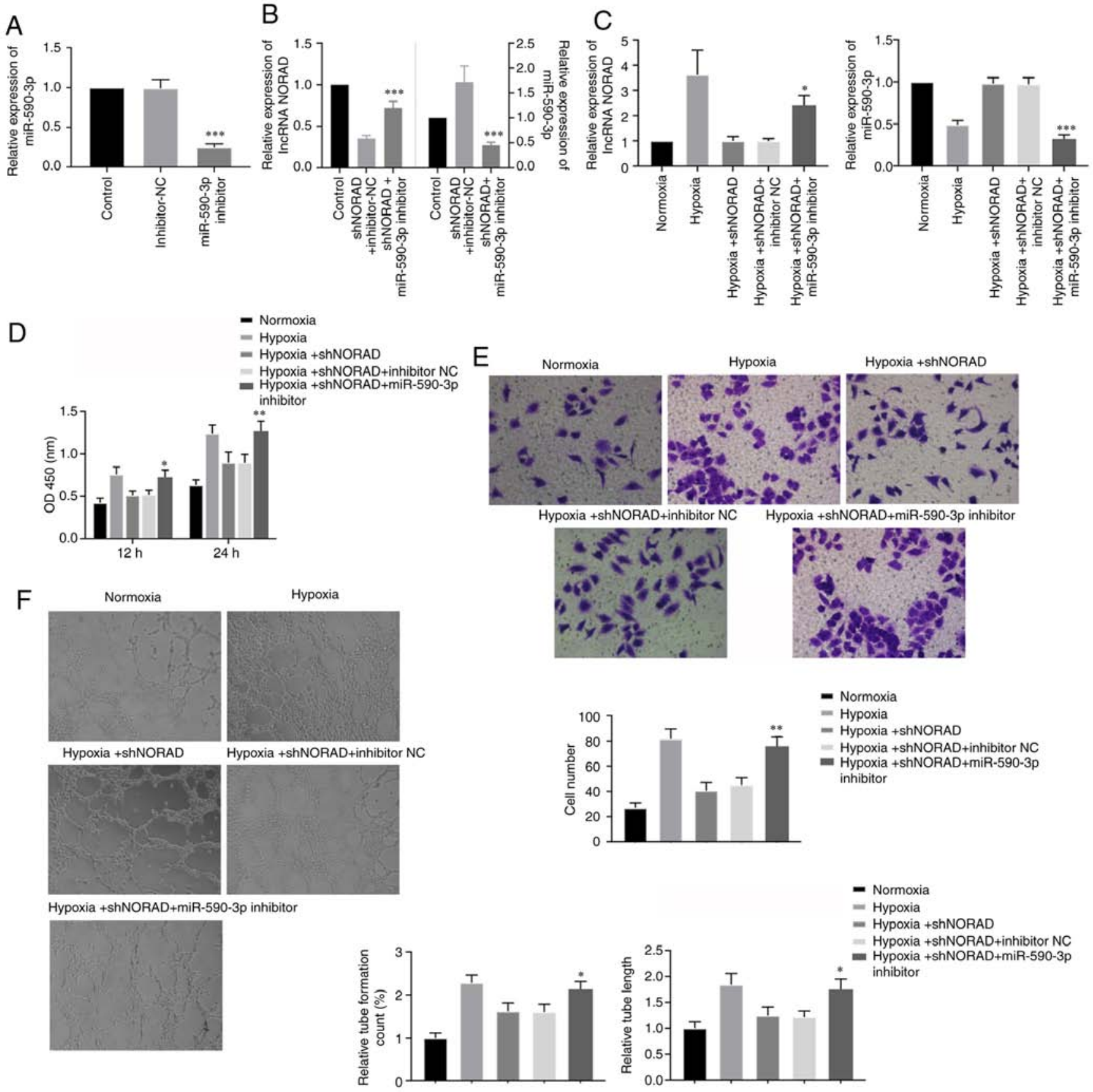

Figure 6. IncRNA NORAD regulates angiogenesis of HUVECs under hypoxia via miR-590-3p. (A) Relative expression of miR-590-3p in HUVECs transfected with miR-590-3p inhibitor and inhibitor-NC. ${ }^{* * *} \mathrm{P}<0.001$ vs. inhibitor-NC. (B) HUVECs were co-transfected with shNORAD and miR-590-3p inhibitor or shNORAD and inhibitor-NC, and expression levels of lncRNA NORAD and miR-590-3p were determined under atmospheric conditions. (C) Expression levels of lncRNA NORAD and miR-590-3p were again assessed under hypoxic condition after the same HUVEC co-transfection. (D) Cell viability, (E) cell migration (magnification, $\mathrm{x} 200$ ) and $(\mathrm{F})$ tube formation (magnification, $\mathrm{x} 100$ ) were measured in HUVECs transfected with both shNORAD and miR-590-3p inhibitor under hypoxia. " $\mathrm{P}<0.05,{ }^{* *} \mathrm{P}<0.01$ and ${ }^{* * *} \mathrm{P}<0.001$ vs. respective inhibitor-NC group. IncRNA, long non-coding RNA; IncRNA NORAD, IncRNA non-coding RNA activated by DNA damage; miR, microRNA; NC, negative control; sh, short hairpin RNA; OD, optical density.

axis under hypoxia, but this was not investigated in the present study. TGF- $\beta$ has been shown to indirectly regulate angiogenesis and also be involved in a miR-590 regulated pathway in cardiac diseases and NORAD-associated functions in lung cancer $(21-23,31,32)$. Thus, TGF- $\beta$ may also affect this NORAD/miR-590-3p regulated hypoxia-induced angiogenesis, but further studies are required to assess this.

The mechanisms regulating angiogenesis are complicated and involve not only the regulation of angiogenic indicators and chemokines, but also the participation of endothelial cells, macrophages or pericytes during various functions of organs under different conditions $(5,12,33)$. Hypoxia-induced diseases are primarily divided into two types: Hypoxia-related ischemic diseases; and angiogenesis-caused cancer types (5). Hypoxia/HIF-1-dependent injuries or angiogenesis are highly associated with ischemic diseases (5). A previous study showed that ischemia/hypoxia-induced angiogenesis briefly improves the blood supply to an ischemic heart, and that this phenomenon is associated with innate immune system activation and macrophage participation in the growing vessels, rather than 

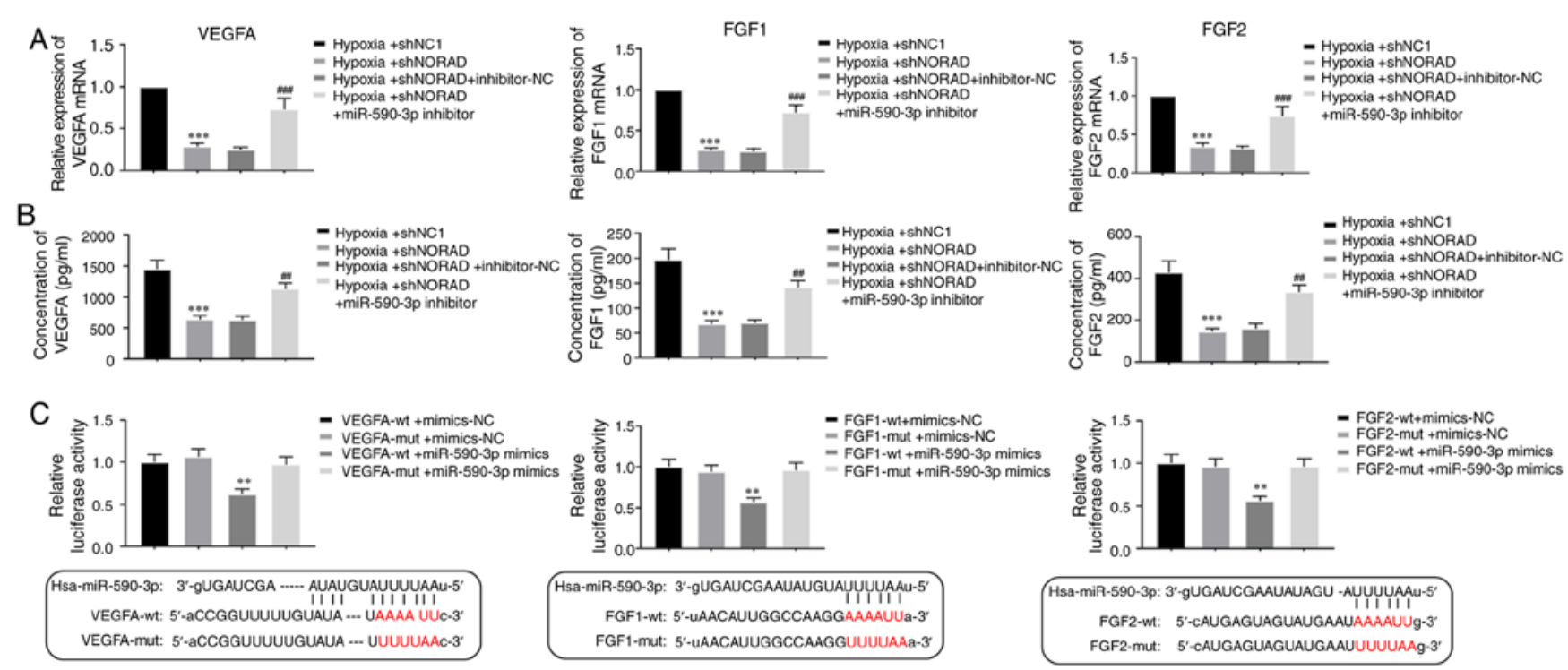

Figure 7. Downstream target genes of the lncRNA NORAD/miR-590-3p axis in HUVECs under hypoxia. (A) Relative mRNA expression levels of VEGFA, FGF1 and FGF2 were identified in HUVECs transfected with shNORAD or shNC1, and co-transfected with shNORAD and miR-590-3p inhibitor, or shNORAD and inhibitor-NC under hypoxia for $24 \mathrm{~h}$. (B) ELISA was performed to measure the concentration of VEGFA, FGF1 and FGF2 in transfected HUVECs under hypoxic condition. ${ }^{* * *} \mathrm{P}<0.001$ in hypoxia + shNORAD vs. hypoxia + shNC1. ${ }^{\# \#} \mathrm{P}<0.01,{ }^{\# \#} \mathrm{P}<0.001$ in hypoxia + shNORAD + miR-590-3p inhibitor vs. hypoxia + shNORAD + inhibitor-NC. (C) Dual luciferase reporter assay was used to assess binding of miR-590-3p with the proangiogenic factors VEGFA, FGF1 and FGF2. Luciferase activity was assessed in 293T cells following co-transfection of miR-590-3p mimics with the 3'UTR of VEGFA, FGF1 and FGF2 or mutant 3'UTRs. ${ }^{* *} \mathrm{P}<0.01$ vs. targeted mutants + miR-590-3p mimics. IncRNA, long non-coding RNA; IncRNA NORAD, IncRNA non-coding RNA activated by DNA damage; miR, microRNA; VEGFA, vascular endothelial growth factor A; FGF, fibroblast growth factor; sh, short hairpin RNA; NC, negative control; 3'UTR, 3' untranslated region; wt, wild-type; mut, mutant.

upregulation of traditional proangiogenic factors (12). However, the detailed mechanisms of this effect have not been determined. Furthermore, a study investigating the angiogenic response under hypoxia in vivo showed that hypoxia-induced angiogenesis of endothelial cells was modulated by a complex network of interactors, and included both the regulation of angiogenic factors such as VEGF, and the regulation of the expression of VEGFRs and VEGF inhibitors (33). In the present study, the regulatory mechanisms of hypoxia-induced angiogenesis in endothelial cells via the NORAD/miR-590-3p axis in vitro were assessed, thus identifying a novel pathway of regulation of angiogenesis. However, in vivo studies are required to confirm the relevance of this axis.

The present study identified the need to use different strategies for treating hypoxia-induced diseases based on the specifics of each case. Therapies targeting a single angiogenic factor, such as VEGF, have been shown to be insufficient, which highlights the requirement for the use of a combination of other drugs to maintain or regulate angiogenesis $(1,5,33)$. Therefore, the hubs of hypoxia-induced angiogenesis should be investigated further. The expression levels of several miRNAs have been shown to be age-associated and cell type-specific $(30,34)$, which needs to be consideration clinically during angiogenic therapy. While there are few previous studies on lncRNAs in hypoxia-induced angiogenic regulation, the present results suggested that IncRNA NORAD could be involved in angiogenic activity of endothelial cells under hypoxic conditions, which may be a potential target for the treatment of associated diseases, such as ischemic heart disease.

In conclusion, the present results indicated that IncRNA NORAD was upregulated, and miR-590-3p was downregulated, in hypoxic HUVECs in vitro and MI left ventricular tissues in vivo. Furthermore, downregulation of lncRNA NORAD in HUVECs decreased cell viability and angiogenic activity, and overexpression of miR-590-3p resulted in similar effects. It was identified that knockdown of both NORAD and miR-590-3p reversed this decrease, and resulted in enhanced angiogenic activity. NORAD was also identified to target miR-590-3p and negatively regulate its expression. It was demonstrated that miR-590-3p further targeted the angiogenic factors VEGFA, FGF1 and FGF2. Therefore, the present results suggested that a lncRNA NORAD/miR-590-3p axis may be involved in the regulation of angiogenesis in HUVECs under hypoxia, which highlights potential targets for treating hypoxia-induced angiogenic diseases.

\section{Acknowledgements}

Not applicable.

\section{Funding}

No funding was received.

\section{Availability of data and materials}

The datasets used and/or analysed during the current study are available from the corresponding author on reasonable request.

\section{Authors' contributions}

$\mathrm{XZ}$ designed the study, performed the experiments, analysed and interpreted the data, and drafted the manuscript. XWe 
and XWa performed the experiments and collected the data. GQ designed and supervised the study, interpreted the data and critically revised the manuscript. All authors read and approved the final manuscript.

\section{Ethics approval and consent to participate}

Animal experiments were performed in accordance with the principles described in the Guide for The Care and Use of Laboratory Animals, and were approved by The Ethics Committee of The First Hospital of China Medical University.

\section{Patient consent for publication}

Not applicable.

\section{Competing interests}

The authors declare that they have no competing interests.

\section{References}

1. Carmeliet P: Mechanisms of angiogenesis and arteriogenesis. Nat Med 6: 389-395, 2000

2. Risau W: Mechanisms of angiogenesis. Nature 386: 671-674, 1997.

3. Yancopoulos GD, Davis S, Gale NW, Rudge JS, Wiegand SJ and Holash J: Vascular-specific growth factors and blood vessel formation. Nature 407: 242-248, 2000.

4. Ferrara N: Vascular endothelial growth factor: Molecular and biological aspects. Curr Top Microbiol Immunol 237: 1-30, 1999

5. Zimna A and Kurpisz M: Hypoxia-inducible factor-1 in physiological and pathophysiological angiogenesis: Applications and therapies. Biomed Res Int 2015: 549412, 2015.

6. Greijer AE, van der Groep P, Kemming D, Shvarts A, Semenza GL, Meijer GA, van de Wiel MA, Belien JA, van Diest PJ and van der Wall E: Up-regulation of gene expression by hypoxia is mediated predominantly by hypoxia-inducible factor 1 (HIF-1). J Pathol 206: 291-304, 2005.

7. Semenza GL: Angiogenesis in ischemic and neoplastic disorders. Annu Rev Med 54: 17-28, 2003.

8. Mori S, Tran V, Nishikawa K, Kaneda T, Hamada Y, Kawaguchi N, Fujita M, Saegusa J, Takada YK, Matsuura N, et al: A dominant-negative FGF1 mutant (the R50E mutant) suppresses tumorigenesis and angiogenesis. PLoS One 8: e57927, 2013.

9. Semenza GL: Oxygen sensing, hypoxia-inducible factors, and disease pathophysiology. Annu Rev Pathol 9: 47-71, 2014.

10. Go AS, Mozaffarian D, Roger VL, Benjamin EJ, Berry JD, Borden WB, Bravata DM, Dai S, Ford ES, Fox CS, et al: Heart disease and stroke statistics-2013 update: A report from the American heart association. Circulation 127: e6-e245, 2013.

11. Murray CJ and Lopez AD: Mortality by cause for eight regions of the world: Global burden of disease study. Lancet 349: 1269-1276, 1997.

12. Lavine KJ, Kovacs A, Weinheimer C and Mann DL: Repetitive myocardial ischemia promotes coronary growth in the adult mammalian heart. J Am Heart Assoc 2: e000343, 2013.

13. Fatica A and Bozzoni I: Long non-coding RNAs: New players in cell differentiation and development. Nat Rev Genet 15: 7-21, 2014.

14. Kung JT, Colognori D and Lee JT: Long noncoding RNAs: Past, present, and future. Genetics 193: 651-669, 2013.

15. Han P, Li W, Lin CH, Yang J, Shang C, Nuernberg ST, Jin KK, $\mathrm{Xu} \mathrm{W}, \mathrm{Lin} \mathrm{CY}, \mathrm{Lin} \mathrm{CJ}$, et al: A long noncoding RNA protects the heart from pathological hypertrophy. Nature 514: 102-106, 2014.
16. Wang K, Long B, Zhou LY, Liu F, Zhou QY, Liu CY, Fan YY and Li PF: CARL IncRNA inhibits anoxia-induced mitochondrial fission and apoptosis in cardiomyocytes by impairing miR-539-dependent PHB2 downregulation. Nat Commun 5: 3596, 2014.

17. Lee S, Kopp F, Chang TC, Sataluri A, Chen B, Sivakumar S, $\mathrm{Yu} \mathrm{H}, \mathrm{Xie} \mathrm{Y}$ and Mendell JT: Noncoding RNA NORAD regulates genomic stability by sequestering PUMILIO proteins. Cell 164: 69-80, 2016.

18. Liu H, Li J, Koirala P, Ding X, Chen B, Wang Y, Wang Z, Wang C, Zhang $\mathrm{X}$ and Mo YY: Long non-coding RNAs as prognostic markers in human breast cancer. Oncotarget 7: 20584-20596, 2016.

19. Li H, Wang X, Wen C, Huo Z, Wang W, Zhan Q, Cheng D, Chen H, Deng X, Peng C and Shen B: Long noncoding RNA NORAD, a novel competing endogenous RNA, enhances the hypoxia-induced epithelial-mesenchymal transition to promote metastasis in pancreatic cancer. Mol Cancer 16: 169, 2017.

20. Miao Z, Guo X and Tian L: The long noncoding RNA NORAD promotes the growth of gastric cancer cells by sponging miR-608. Gene 687: 116-124, 2019.

21. Kawasaki N, Miwa T, Hokari S, Sakurai T, Ohmori K, Miyauchi K, Miyazono K and Koinuma D: Long noncoding RNA NORAD regulates transforming growth factor- $\beta$ signaling and epithelial-to-mesenchymal transition-like phenotype. Cancer Sci 109: 2211-2220, 2018

22. Ekhteraei-Tousi S, Mohammad-Soltani B, Sadeghizadeh M, Mowla SJ, Parsi S and Soleimani M: Inhibitory effect of hsa-miR-590-5p on cardiosphere-derived stem cells differentiation through downregulation of TGF $\beta$ signaling. J Cell Biochem 116: 179-191, 2015.

23. Jafarzadeh M and Soltani BM: Hsa-miR-590-5p interaction with SMAD3 transcript supports its regulatory effect on The TGF $\beta$ signaling pathway. Cell J 18: 7-12, 2016.

24. Nallamshetty S, Chan SY and Loscalzo J: Hypoxia: A master regulator of microRNA biogenesis and activity. Free Radic Biol Med 64: 20-30, 2013.

25. Kumar MM and Goyal R: LncRNA as a therapeutic target for angiogenesis. Curr Top Med Chem 17: 1750-1757, 2017.

26. Rehmsmeier M, Steffen P, Hochsmann M and Giegerich R: Fast and effective prediction of microRNA/target duplexes. RNA 10: $1507-1517,2004$

27. Livak KJ and Schmittgen TD: Analysis of relative gene expression data using real-time quantitative PCR and the 2(-Delta Delta C(T)) method. Methods 25: 402-408, 2001.

28. Guide for the Care and Use of Laboratory Animals. Institute for Laboratory Animal Research. National Academies Press, Washington, DC, 2011.

29. Yang Z, Zhao Y, Lin G, Zhou X, Jiang X and Zhao H: Noncoding RNA activated by DNA damage (NORAD): Biologic function and mechanisms in human cancers. Clin Chim Acta 489: 5-9, 2019.

30. Madanecki P, Kapoor N, Bebok Z, Ochocka R, Collawn JF and Bartoszewski R: Regulation of angiogenesis by hypoxia: The role of microRNA. Cell Mol Biol Lett 18: 47-57, 2013.

31. Folkman J and Shing Y: Angiogenesis. J Biol Chem 267: 10931-10934, 1992.

32. Geng L, Chaudhuri A, Talmon G, Wisecarver JL and Wang J: TGF-Beta suppresses VEGFA-mediated angiogenesis in colon cancer metastasis. PLoS One 8: e59918, 2013.

33. Aplin AC and Nicosia RF: Hypoxia paradoxically inhibits the angiogenic response of isolated vessel explants while inducing overexpression of vascular endothelial growth factor. Angiogenesis 19: 133-146, 2016.

34. Noren Hooten N, Abdelmohsen K, Gorospe M, Ejiogu N, Zonderman $\mathrm{AB}$ and Evans MK: microRNA expression patterns reveal differential expression of target genes with age. PLoS One 5: e10724, 2010.

This work is licensed under a Creative Commons Attribution-NonCommercial-NoDerivatives 4.0 International (CC BY-NC-ND 4.0) License. 